M. Stöhr, I. Boxx, C. Carter and W. Meier, Dynamics of lean blowout of a swirl-stabilized flame in a gas turbine model combustor, Proceedings of the Combustion Institute 33 (2011) 2953-2960.

The original publication is available at www.elsevier.com

http://dx.doi.org/10.1016/j.proci.2010.06.103 


\title{
Dynamics of lean blowout of a swirl-stabilized flame in a gas turbine model combustor
}

\author{
M. Stöhr ${ }^{a, *}$, I. Boxx $^{\text {a }}$, C. Carter $^{b}$, W. Meier ${ }^{a}$ \\ ${ }^{a}$ German Aerospace Center (DLR), Institute of Combustion Technology, \\ Pfaffenwaldring 38-40, 70569 Stuttgart, Germany \\ ${ }^{\mathrm{b}}$ Air Force Research Laboratory, Wright-Patterson Air Force Base, Dayton, \\ $\mathrm{OH} 45433$, USA
}

\begin{abstract}
Lean blowout (LBO) of a partially premixed swirl flame is studied using chemiluminescence imaging and simultaneous stereo-PIV and OH-PLIF measurements at repetition rates up to $5 \mathrm{kHz}$. The flame, which is operated with methane and air in a gas turbine model combustor at atmospheric pressure, features a pronounced precessing vortex core (PVC) at the inner shear layer. In the first part of the study, the stabilization mechanism of the flame close to LBO is investigated. The fields of velocity and $\mathrm{OH}$ show that near $\mathrm{LBO}$ there are essentially two regions where reaction takes place, namely the helical zone along the PVC and the flame root around the lower stagnation point. The zone along the PVC is favorable to the flame due to low strain rates in the vortex center and accelerated mixing of burned and fresh gas. The flame root, which is located close to the nozzle exit, is characterized by an opposed flow of hot burned gas and relatively fuel-rich fresh gas. Due to the presence of high strain rates, the flame root is inherently unstable near LBO, featuring frequent extinction and re-ignition. The blowout process, discussed in the second part of the study, starts when the extinction of the flame root persists over a critical length of time. Subsequently, the reaction in the helical zone can no longer be sustained and the flame finally blows out. The results highlight the crucial role of the flame root, and suggest that well-aimed modifications of flow field or mixture fraction in this region might shift the LBO limit to leaner conditions.
\end{abstract}

Key words: Lean blowout, Swirl-stabilized flame, Precessing vortex core, Gas turbine combustion, High-speed laser diagnostics

* Corresponding author. Fax: +49 711 6862578, Email: michael.stoehr@dlr.de 


\section{Introduction}

Due to stringent emission requirements, modern gas turbine (GT) combustors work under lean conditions, which lead to lower flame temperatures and therefore reduced $\mathrm{NO}_{x}$ emissions. The operation of a combustor close to the lean blowout (LBO) limit increases the risk of a complete flame blowout. Blowout poses a significant safety hazard when occuring in aircraft engines and requires an expensive shutdown and restart procedure in land-based engines for power generation.

Flames in GT combustors are most often stabilized by inducing a swirling flow of the reactants. Vortex breakdown of the swirling flow and cross-section extension at the nozzle exit lead to the formation of inner and outer recirculation zones. These zones transport hot combustion products back to the nozzle, which enhances the ignition of unburned gas and thereby stabilizes the flame. The mechanisms that lead to LBO in turbulent swirl-stabilized flames, however, are poorly understood owing to the complex interactions of chemistry, flow and transport. Previous studies of LBO dynamics have mainly focused on bluff-body stabilized flames as summarized in a recent review by Shanbhogue et al. [1]. The LBO limit of swirl flames is therefore difficult to predict, and has to be determined experimentally. It was found that the LBO limit depends on aerodynamical parameters including swirl number [2-4] and sense of secondary swirl [2]. Furthermore the LBO limit can be extended to lower equivalence ratios through the injection of pilot fuel/air [5], addition of hydrogen [6-8] or an increase of preheat temperature [2,9], whereas the influence of pressure has been found to be small [7]. During operation, the proximity to LBO is currently determined on the basis of empirical quantities, which are mostly based on the intensity of low-frequency combustion oscillations [10-13] and require careful calibration before operation.

The objectives to extend the LBO limit to lower equivalence ratios, and to reliably determine the proximity to LBO during operation motivate a desire for a better understanding of swirl flame dynamics near the LBO limit. The recently available high-speed laser diagnostic systems are a promising tool for the investigation of transient combustion phenomena [14,15], but only few reports of their application to the LBO of swirl flames exist. Using chemiluminescence imaging and laser scattering at $1.9 \mathrm{kHz}$, Muruganandam and Seitzman found that the presence of cold reactants in the inner recirculation zone triggers the extinction process in a premixed swirled combustor [16]. The objective of the present study is to contribute to an improved understanding of the mechanisms of LBO in GT-typical swirl flames. The dynamics of a partially premixed, swirl-stabilized flame in a gas turbine model combustor is investigated shortly before and during LBO events using chemiluminescence imaging and simultaneous stereoscopic particle image velocimetry (PIV) and 
planar laser-induced fluorescence (PLIF) of $\mathrm{OH}$ at repetition rates up to 5 $\mathrm{kHz}$. In the first part of the study, the stabilization mechanism of a flame operated close to the LBO limit is characterized, including a discussion of the role of a precessing vortex core (PVC). In the second part, the failure in this mechanism that leads to LBO is investigated.

\section{Experimental setup}

\subsection{Combustor and flame}

A schematic of the gas turbine model combustor is shown in Fig. 1. Dry air at room temperature enters a plenum (diameter $79 \mathrm{~mm}$, height $65 \mathrm{~mm}$ ) and then separately passes two radial swirl generators. The two co-swirling flows enter the combustion chamber through a central nozzle (diameter $15 \mathrm{~mm}$ ) and an annular nozzle (inner diameter $17 \mathrm{~mm}$, outer diameter $25 \mathrm{~mm}$ contoured to an outer diameter of $40 \mathrm{~mm}$ ). Non-swirling $\mathrm{CH}_{4}$ is fed through 72 channels $\left(0.5 \times 0.5 \mathrm{~mm}^{2}\right)$ forming a ring between the air nozzles. The exit planes of the fuel and central air nozzles are located $4.5 \mathrm{~mm}$ below the exit plane of the outer air nozzle which is taken as height $y=0$. The combustion chamber has a square cross section of $85 \times 85 \mathrm{~mm}^{2}$, a height of $114 \mathrm{~mm}$, and is enclosed by 4 quartz windows held by 4 posts (diameter $10 \mathrm{~mm}$ ) in the corners allowing very good optical access. A conical top plate with a central exhaust tube (diameter $40 \mathrm{~mm}$, length $50 \mathrm{~mm}$ ) forms the exit.

The measurements in this study were performed with the combustor operating at a thermal power of $P=7.6 \mathrm{~kW}$ and a global equivalence ratio of $\phi=0.55$. The swirl number $S$ has been estimated from LDV measurements as $S \approx 0.55$, and

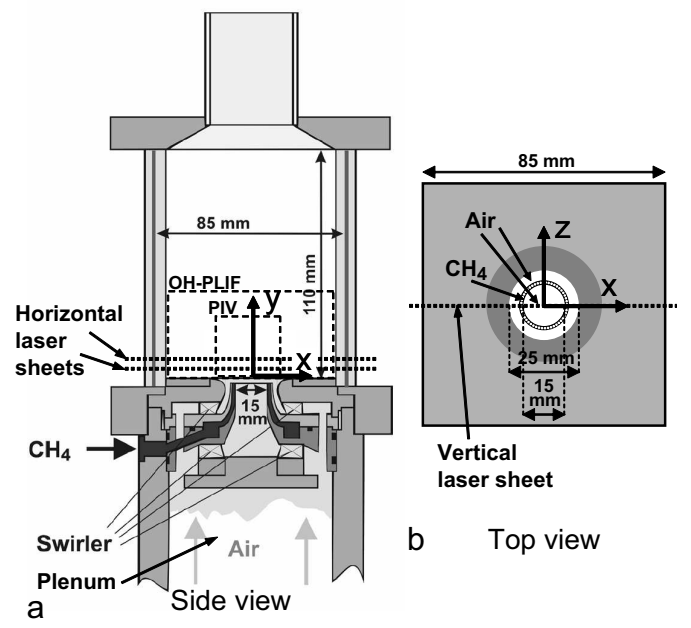

Fig. 1. (a) Side view and (b) top view of the gas turbine model combustor. 
the Reynolds number of the cold air as Re $\approx 15000[17,18]$. At this condition, phases of stable operation are intermittently interrupted by transient events of blowout and reignition, which appear randomly in time without any previous changes of the mean flame shape. During these events the flame completely extinguishes in the combustion chamber, moves downstream and stabilizes in the exhaust tube. From there hot burned gas is recirculated to the burner nozzle, and, about $100 \mathrm{~ms}$ later, the flame reignites above the nozzle and returns to stable operation until the next event. The average duration of stable operation between the transient events is around 1-2 s and decreases when $\phi$ is decreased slightly. As $\phi$ is decreased further, reignition does not occur anymore, and the flame ultimately blows out.

\subsection{Measuring techniques}

The diagnostic techniques used in this study have already been detailed in previous publications and therefore only a brief description will be provided here. The main part of the measurements was performed with a combined stereoPIV and OH-PLIF system operated at a repetition rate of $5 \mathrm{kHz}$, which is identical to the system used in [15]. The stereo-PIV system is based on a dual-cavity Nd:YAG laser (Edgewave IS-611DE, $2.6 \mathrm{~mJ} /$ pulse at $532 \mathrm{~nm}$ after SHG) and a pair of CMOS cameras (LaVision HSS5). The OH-PLIF system consists of a dye laser (Sirah Cobra-Stretch HRR, $100 \mu \mathrm{J} /$ pulse at $283.2 \mathrm{~nm}$ after SHG) pumped with a Nd:YLF laser (Edgewave IS-811E, $3.8 \mathrm{~mJ} /$ pulse at $523 \mathrm{~nm}$ after SHG) and an intensified CMOS camera (LaVision HSS6). The beams of the two laser systems are expanded into two coplanar vertical light sheets across the central section $(z=0)$ of the combustion chamber (Fig. 1b). While the field of view for the stereo-PIV was limited to a $30 \times 30 \mathrm{~mm}^{2}$ region above the nozzle, the OH-PLIF field extends across the full width and up to a height of $y=45 \mathrm{~mm}$ (Fig. 1a). Chemiluminescence image sequences were recorded using the OH-PLIF camera at $5 \mathrm{kHz}$ with an exposure time (intensifier gate width) of $20 \mu \mathrm{s}$. All cameras had sufficient on-board memory for at least 4096 individual recordings corresponding to an aquisition time of $0.8 \mathrm{~s}$. The combination of high repetition rate and long record length enables detailed insights into transient phenomena like blowout, which could not easily be studied by other methods. A second stereo-PIV and OH-PLIF system with a repetition rate of $5 \mathrm{~Hz}$ and pulse energies of $2 \times 120 \mathrm{~mJ}$ and $2.3 \mathrm{~mJ}$, respectively, was used for measurements in horizontal sections at $y=5 \mathrm{~mm}$ and $y=10 \mathrm{~mm}$ (Fig. 1a). This system is identical to the one used in [19]. 


\section{Stabilization mechanism close to LBO}

\subsection{Average flame shape, flow field and thermochemical state}

Figure 2 shows the average $\mathrm{OH}^{*}$ chemiluminescence and velocity field during a period of stable operation before a blowout event. The flame shape and flow field are very similar to fully stable operation (without intermediate blowout events) at slightly higher $\phi$. The $\mathrm{OH}^{*}$ signal is used as a marker of the location of heat release [20]. It shows that the heat release zone lies between $y=5 \mathrm{~mm}$ and $y=40 \mathrm{~mm}$ in axial direction and within a radius of $r<35 \mathrm{~mm}$ from the axis. The flow field is typical of confined swirl flames, consisting of a coneshaped stream of fresh gas entering the chamber from the burner nozzle, an inner recirculation zone (IRZ) and an outer recirculation zone (ORZ). Strong velocity gradients occur in the inner shear layer (ISL) between the inflow and the IRZ, and in the outer shear layer (OSL) between inflow and ORZ. As it will be shown below, a coherent helical vortex, the so-called precessing vortex core (PVC), is located in the ISL.

Distributions of average mixture fraction and temperature, which were measured in a previous study using Raman spectroscopy $[17,18]$, are shown in Fig. 3. The mixture fraction $f$ is the mass fraction in the measurement vol-

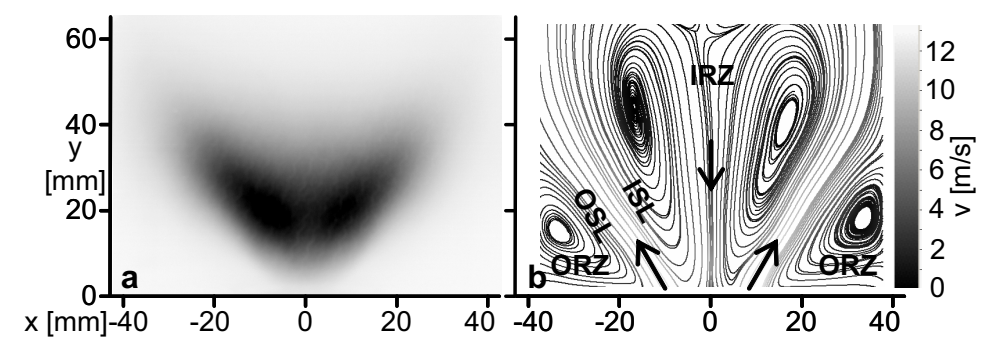

Fig. 2. Averages of (a) $\mathrm{OH}^{*}$ chemiluminescence and (b) velocity field.

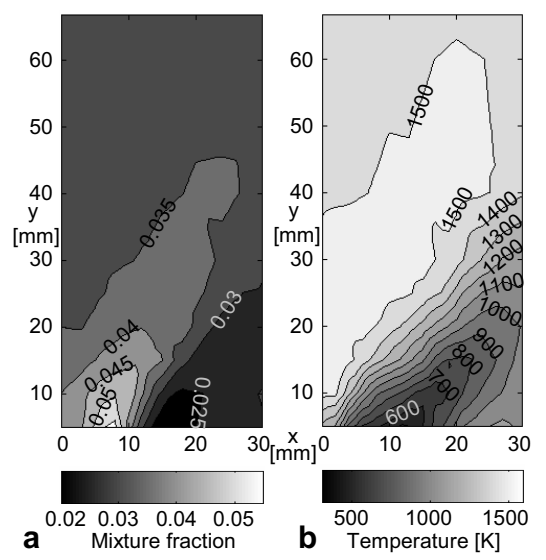

Fig. 3. Distributions of (a) mixture fraction and (b) temperature. 
ume originating from the fuel nozzle. For methane and air, the stoichiometric value is $f_{\text {stoich }}=0.055$, and the value for $\phi_{\text {global }}=0.55$ is $f_{\text {global }}=0.031$. It is seen that in the IRZ the gas composition is relatively fuel rich $\left(f>f_{\text {global }}\right)$, whereas in the ORZ the composition is relatively lean $\left(f<f_{\text {global }}\right)$. This is caused by the location of the fuel nozzles at $r=8 \mathrm{~mm}$. The effect is strongest at $y=5 \mathrm{~mm}$ (measurements were restricted to $y \geq 5 \mathrm{~mm}$ ), where values up to $f=0.056$ are encountered at $x=8 \mathrm{~mm}$, and decreases downstream due to mixing progress.

Figure $3 \mathrm{~b}$ shows the temperature increase within the jet flow region from $T=600 \mathrm{~K}$ at $y=5 \mathrm{~mm}$ to $T=1400 \mathrm{~K}$ at $y=40 \mathrm{~mm}$ as a result of reaction progress. The temperatures in the IRZ are significantly higher than those in the ORZ, which results from the relatively rich and lean conditions, respectively. For a more detailed discussion of the thermochemical state of the flame, we refer to $[17,18]$.

\subsection{Helical flame zone}

Figure 4 shows an instantaneous $\mathrm{OH}^{*}$ chemiluminescence image during stable operation. The heat release zone resembles a $2 \mathrm{D}$ projection of a helical structure, marked by the dashed line. Previous experimental studies on the same combustor have identfied a helical vortex, a so-called precessing vortex core (PVC), located at the ISL in the flame $[19,15]$. The presence of a PVC has also been seen in a large eddy simulation (LES) of the same operating condition as in this work [22]. Taken together, these findings indicate that the heat release of the flame is at least partially aligned with the PVC.

This phenomenon is further illustrated by the time-series of simultaneous PIV and OH-PLIF measurements displayed in Fig. 5. The velocity field shows a zig-zag arrangement of vortices, marked by arrows, moving downstream along the ISL. The zig-zag arrangement in the $2 \mathrm{D}$ cross-section is a representation of the 3D helical PVC, and the downstream motion of the vortices represents the rotation of the PVC around the central axis. From a Fourier analysis of

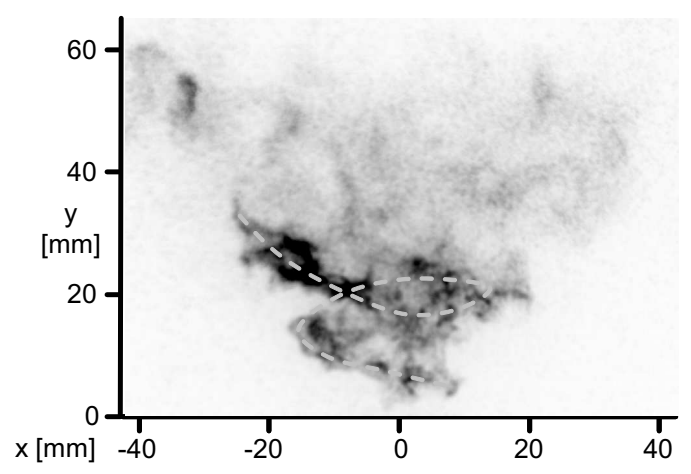

Fig. 4. Instantaneous $\mathrm{OH}^{*}$ chemiluminescence image. 


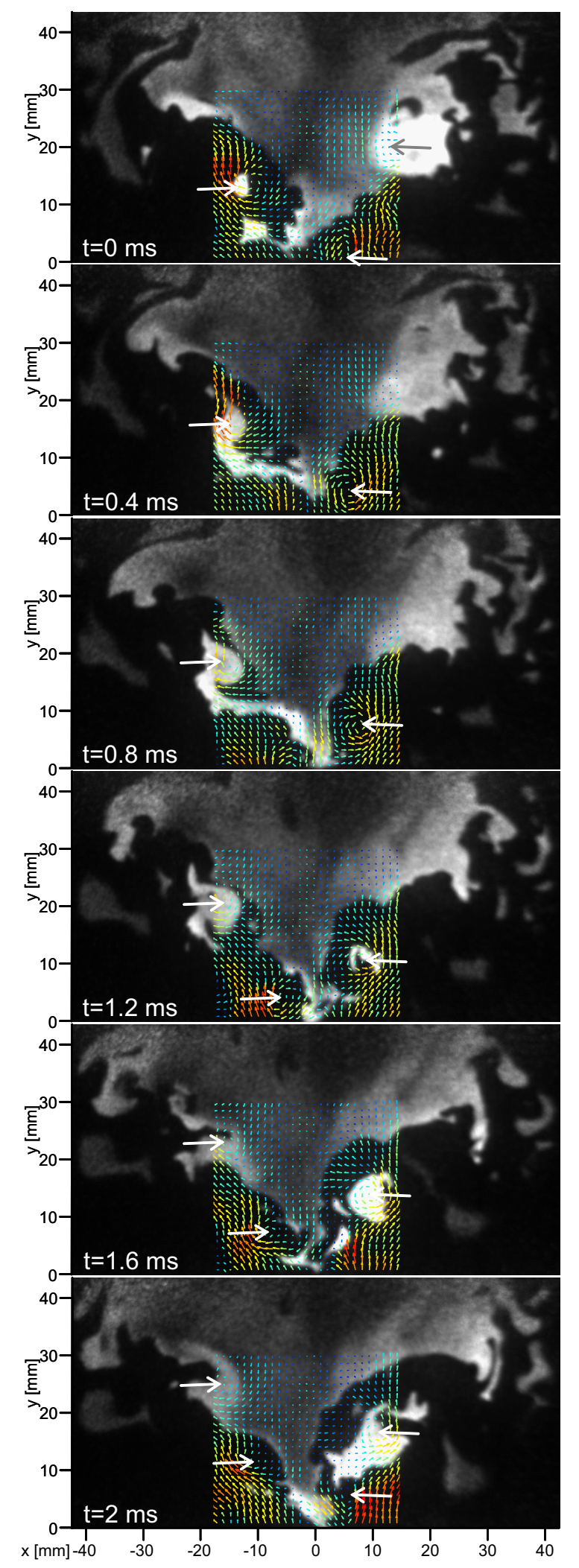

Fig. 5. Time series of simultaneous PIV and OH-PLIF measurements showing the stabilized flame close to LBO. Vortex centers are marked by arrows. The vector color scale is the same as in Fig. 6. 


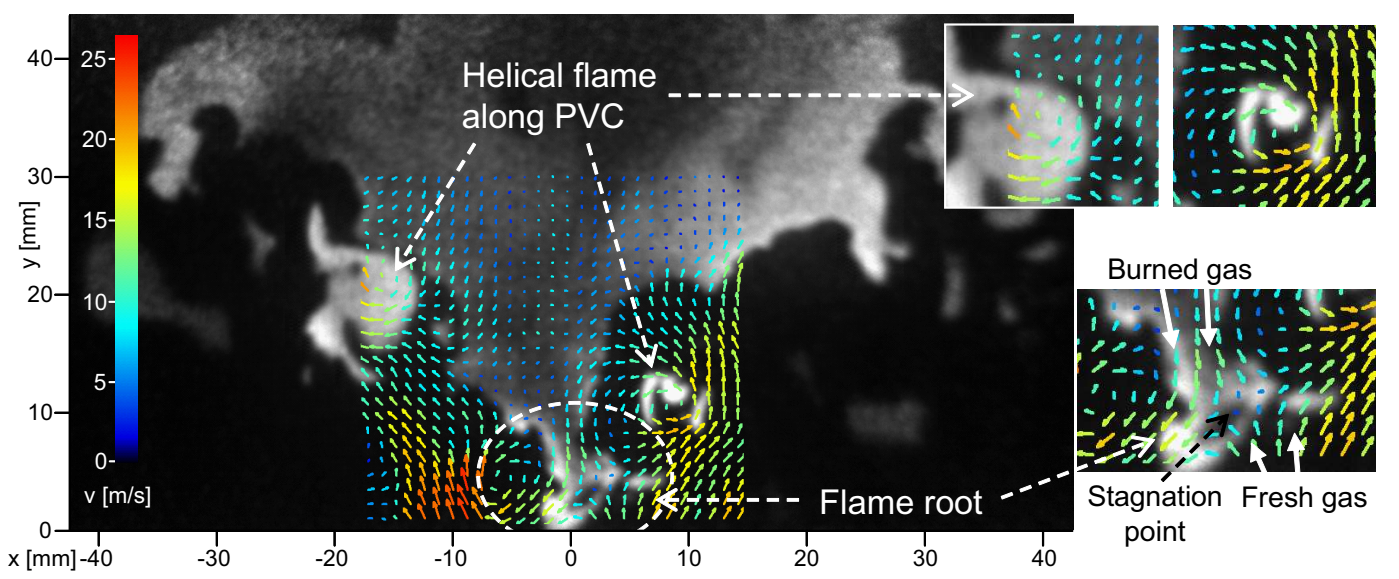

Fig. 6. Enlarged version of Fig. $5(\mathrm{t}=1.2 \mathrm{~ms})$, highlighting the flame zones in the vortex centers and at the flame root. Vector colors represent the absolute value of velocity.

the velocities in the ISL, the frequency of the PVC is determined as $510 \mathrm{~Hz}$.

The corresponding flame structures are characterized by the grayscale $\mathrm{OH}-$ PLIF images. Regions with high levels of $\mathrm{OH}$ (light gray to white) indicate superequilibrium $\mathrm{OH}$, which is formed in the reaction zones and has a half-life period of $\approx 1$ ms under atmospheric pressure [21]. Medium and low levels of $\mathrm{OH}$ (medium and dark gray) represent burned gas whose $\mathrm{OH}$ concentration has decayed towards equilibrium while it was transported away from the reaction zone. The equilibrium $\mathrm{OH}$ concentration increases with temperature and as the stoichiometric condition is approached [21]. Regions without $\mathrm{OH}$ (black) represent gas with low or medium temperature $(T<1500 \mathrm{~K})$, i.e., unburned gas with possible admixture of burned gas.

In the OH-PLIF images, the major part of $\mathrm{OH}$ is found in the IRZ and ISL. This is caused by the higher temperatures and the relatively fuel rich gas in the IRZ as pointed out in Section 3.1. High OH levels indicating reaction zones are often found close to the vortex centers, which is highlighted in the enlarged views in Fig. 6. This further confirms the above finding that reaction and heat release largely take place along the PVC. The reaction zones appear first as small pockets of $\mathrm{OH}$ as seen at $t=0 \mathrm{~ms}$ and $t=1.2 \mathrm{~ms}$ on the left- and righthand side, respectively. Then the reaction propagates away from the vortex center while the vortex moves downstream.

To help understand the favored location of the flame near the PVC, extinction limits and stretch rates are discussed below. The mechanisms of extinction in turbulent, non- or partially premixed flames are generally still an active field of research owing to the complex effects of scalar dissipation [23], unsteady strain rate [24] and equivalence ratio fluctuations [25]. A quantification of these effects would require simultaneous measurements of (at least) mixture fraction, temperature and flow field, and is therefore beyond the scope of 
this work. Nevertheless, a recent study of near-LBO dynamics of a bluff-body stabilized flame [26] has demonstrated that the steady extinction strain rate of premixed flames gives a useful indication for the stretch sensitivity of the flame as the LBO limit is approached, and we will use it for comparison with measured strain rates in the following.

Several experimental and numerical studies have shown that the stretch extinction limit of lean premixed methane-air flames decreases considerably when the equivalence ratio is reduced $[27,28]$. For a premixed opposed jet flame, $\mathrm{Ju}$ et al. found a decrease of the extinction stretch rate from $\approx 1800 \mathrm{~s}^{-1}$ at $\phi=0.75$ to $\approx 460 \mathrm{~s}^{-1}$ at $\phi=0.55$ [28]. Principal strain rates were determined for the present flow fields, and the lowest absolute values $\left(<500 \mathrm{~s}^{-1}\right)$ were found in the vortex centers, where solid-body rotation occurs. In most other parts of the ISL and IRZ, the values are higher than $1000 \mathrm{~s}^{-1}$. The decrease of the extinction stretch rate can therefore account for the alignment of the flame zone with the PVC at the equivalence ratio of $\phi=0.55$. A second effect that favors this alignment is the location of the PVC between the stream of unburned gas and the recirculating burned gas. The inner vortex core featuring solid-body rotation is surrounded by a zone where velocities decrease with distance from the center. The decrease of velocity implies high strain rates, leading to accelerated mixing $[29,30]$ and thereby to a supply of heat and radicals to the unburned gas.

\subsection{Flame root}

Besides the helical flame zone, there is a second zone where reaction takes place, namely the flame root located directly above the nozzle. As shown in Fig. 6, the flame root is located around the instantaneous stagnation point where fresh gas from the nozzle and burned gas from the IRZ collide frontally. In contrast to the helical flame zone, strain rates are very high in this region. In the example shown in Fig. 6, the 2D principal strain rates at the stagnation point were determined as $5620 \mathrm{~s}^{-1}$ (extensive) and $4058 \mathrm{~s}^{-1}$ (compressive). These values are well above the stretch extinction limit of $\approx 460 \mathrm{~s}^{-1}$ at $\phi=0.55$ [28]. The flame root can, to some extent, withstand the high strain rates because of the locally relatively fuel rich and hot conditions (see Fig. 3 and Section 3.1), which imply a higher extinction limit. Also, the frontal collision with burned gas from the IRZ leads to an efficient supply of heat and radicals to the fresh gas. Nevertheless, the flame root is highly susceptible to stretch extinction due to the high strain rates, especially at lean conditions. In Fig. 6 it can be seen that right at the stagnation point the $\mathrm{OH}$ concentration has decreased, which is an indication for local extinction. This agrees with results of Böhm et al., who found that extinction in turbulent opposed jet flames starts at the stagnation point [14]. Local extinction in the flame root region 


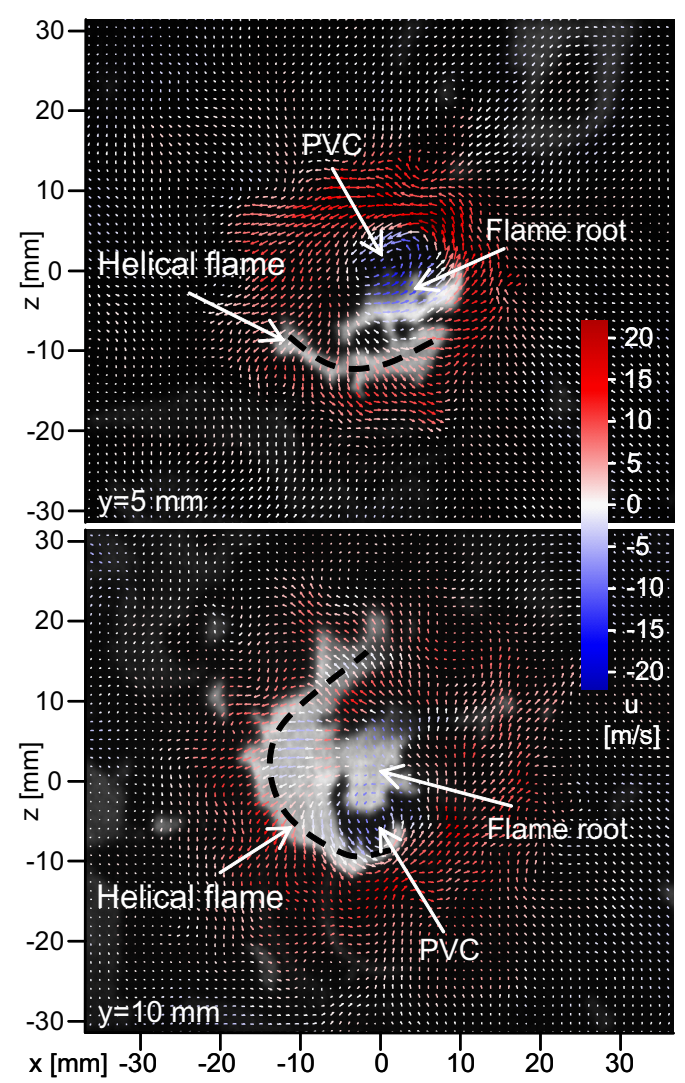

Fig. 7. Simultaneous PIV and OH-PLIF measurements in horizontal sections at (a) $y=5 \mathrm{~mm}$ and (b) $y=10 \mathrm{~mm}$. Vector colors represent axial velocity.

has also been found in two previous studies of the same combustor. Using Raman spectroscopy, Meier et al. found a large number of partially reacted mixtures in this region and concluded that these are an indication for local extinction [18]. A time-resolved visualization of local extinction in the flame root region using high-speed laser diagnostics has been reported by Boxx et al. [15]. The latter article also provides a more detailed discussion of extinction mechanisms in this region, which is beyond the scope of this study.

The partitioning of the flame into an approximately helical flame zone and a flame root can also be seen in the measurements in the horizontal sections shown in Fig. 7. The helical flame appears as a crescent-shaped region along the ISL, and the flame root is located in the IRZ. It can further be seen that the two regions are at least temporarily connected. A similar connection also appears in the time-resolved sequence in the vertical section (Fig. 5) on the left-hand side at $t=0.4 \mathrm{~ms}$ and $t=0.8 \mathrm{~ms}$. The disappearance of the connection at $t=1.2 \mathrm{~ms}$ may represent either a disruption or an out-of-plane displacement of the connection.

The observed phenomena indicate that there is a mutual dependence of the two flame zones. The flame root essentially requires the upstream flow of hot 


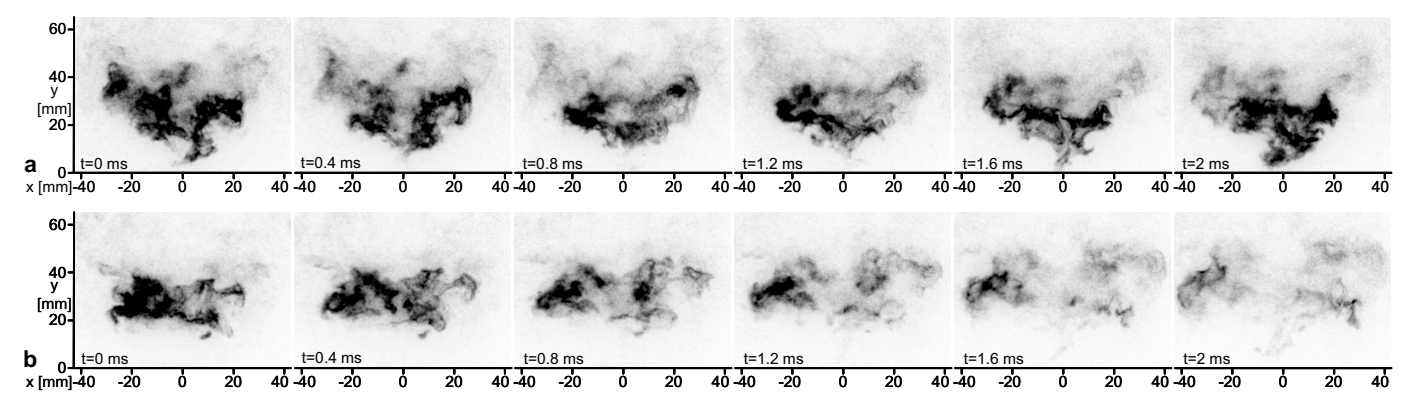

Fig. 8. Time series of $\mathrm{OH}^{*}$ chemiluminescence. (a) extinction and reignition of flame root. (b) ultimate blowout event.

exhaust gas in the IRZ coming from the helical zone. On the other hand, the flame root is a source of heat and radicals close to the upstream end of the helical zone, and thereby contributes to the ignition of the fresh gas in the helical zone.

\section{Onset and progress of blowout}

As described in Section 3.3, the flame root is subject to high strain rates and therefore susceptible to stretch extinction especially at lean conditions. Fig. 8a shows a sequence of chemiluminescence images during stable operation where the flame root extinguishes $(t=0.8 \mathrm{~ms})$ and reignites shortly afterwards $(t=2$ $m s)$. Such precursive events are often seen during stable operation, demonstrating the fragility of the flame root, but also its ability to reestablish, e.g., owing to flame propagation from the helical zone.

Due to intermittent fluctuations of strain rate or equivalence ratio, the reignition of the flame root may be inhibited. This happens during the ultimate LBO event shown in Fig. 8b. At $t=0 \mathrm{~ms}$ the flame root has already extinguished. Reignition does not occur, and subsequently, starting from $t=0.8 \mathrm{~ms}$, the helical flame zone also extinguishes.

Figure 9 shows a time-series of PIV and OH-PLIF measurements of a similar LBO event. At $t=0 \mathrm{~ms}$ the flame root is extinguished but the helical flame is still present. In contrast to the stable condition where the flame root is active (Fig. 5), now the ignition at the vortex centers does not occur, and subsequently ultimate blowout occurs. The results above demonstrate that the supply of heat and radicals by the flame root plays an important role for flame stabilization. An analysis of several LBO events (11 events were recorded with simultaneous PIV and OH-PLIF) shows that during these events, the flame root extinguishes first. If this extinction persists longer than around $2 \mathrm{~ms}$ (corresponding to one rotation of the PVC), the whole flame will blow out. While the fragility of the flame root generally arises from the combination of 


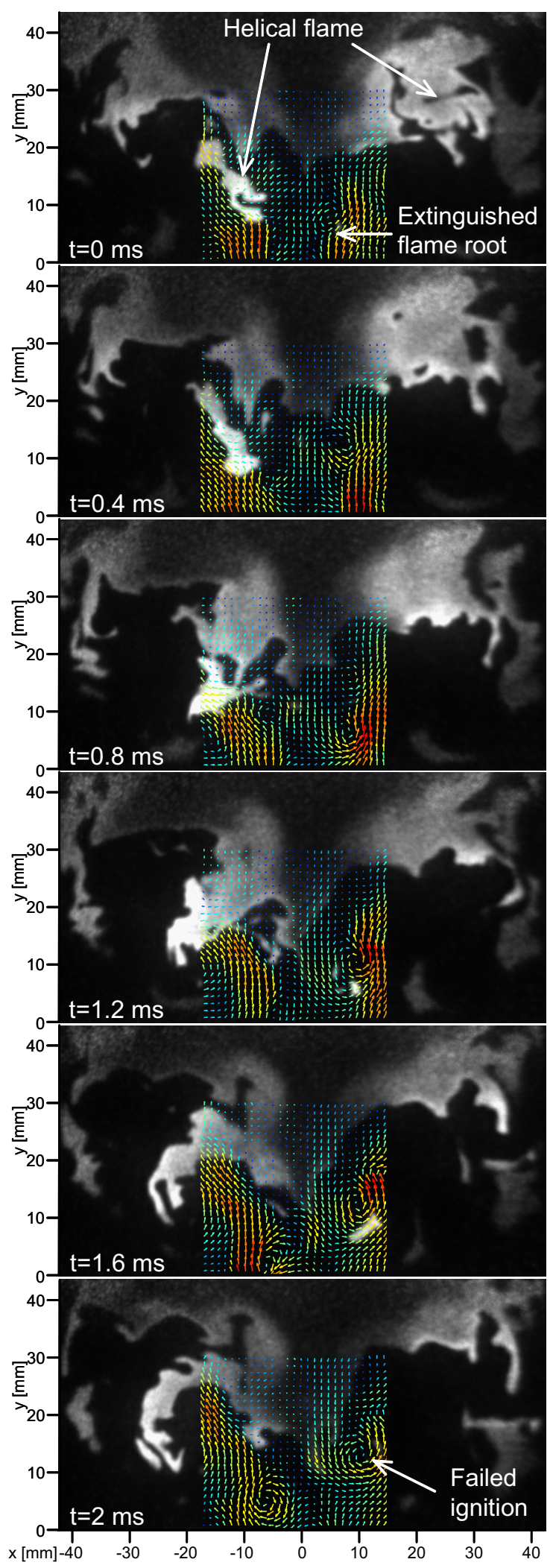

Fig. 9. Time series of simultaneous PIV and OH-PLIF measurements during a lean blowout event. The vector color scale is the same as in Fig. 6 . 
high strain rates and low stretch extinction limit, there are several distinct processes that can finally trigger the extinction, including entrainment of cold unburned gas into the IRZ or intermittent variations of strain rate or mixture fraction. As the flame root is generally close to extinction, even a minor manifestation of such a process can trigger extinction.

The results suggest that well-aimed modifications of the flow field or mixture fraction in this region might shift the LBO limit to leaner conditions. This is consistent with the study of Muruganandam et al., who found that injection of a premixed pilot fuel/air flow into the center of the inlet was able to avoid blowout of a swirl-stabilized flame [5].

\section{$5 \quad$ Summary and Conclusions}

The dynamics of a partially premixed, swirl-stabilized flame in a gas turbine model combustor operated close to the LBO limit was investigated using chemiluminescence imaging and simultaneous stereo-PIV and OH-PLIF measurements at repetition rates up to $5 \mathrm{kHz}$. The fields of velocity and $\mathrm{OH}$ showed that close to the LBO limit, there are essentially two regions where reaction takes place. One of them is the helical zone along a PVC at the ISL, which is favorable to the flame due to low strain rates and accelerated mixing of burned and fresh gas. The other region is the flame root around the lower stagnation point, which is characterized by an opposed flow of hot burned gas and relatively fuel-rich fresh gas. It was found that due to the presence of high strain rates, the flame root is inherently unstable near LBO, featuring frequent extinction and re-ignition. The blowout process starts when the extinction of the flame root exceeds a critical length of time, which was found to be about $2 \mathrm{~ms}$ (equalling the period for the PVC oscillation). Then, the reaction in the helical zone can no longer be sustained, and the flame finally blows out. The results highlight the important role of the flame root and suggest that wellaimed modifications of the flow field or mixture fraction in this region might shift the LBO limit to leaner conditions.

\section{Acknowledgments}

The contribution of R. Sadanandan to the OH-PLIF measurements in the horizontal sections is gratefully acknowledged. 


\section{References}

[1] S. J. Shanbhogue, S. Husain, T. Lieuwen, Prog. Energy Combust. Sci. 35 (2009) 98120

[2] A. Ateshkadi, V. G. McDonell, G. S. Samuelsen, Proc. Combust. Inst. 28 (2000) $1281-1288$

[3] M. R. Johnson, D. Littlejohn, W. A. Nazeer, K. O. Smith, R. K. Cheng, Proc. Combust. Inst. 30 (2005) 2867-2874

[4] K. Kumaran, U. S. P. Shet, Combust. Flame 151 (2007) 391-395

[5] T. M. Muruganandam, S. Nair, D. Scarborough, Y. Neumeier, J. Jagoda, T. Lieuwen, J. Seitzman, B. Zinn, J. Prop. Power 21 (2005) 807-814

[6] Q. Zhang, D. R. Noble, S. J. Shanbhogue, T. Lieuwen, Proceedings of ASME Turbo Expo, Montreal, 2007, GT2007-27308

[7] P. Strakey, T. Sidwell and J. Ontko, Proc. Combust. Inst. 31 (2007) 3173-3180

[8] D. M. Wicksall, A. K. Agrawal, R. W. Schefer, J. O. Keller, Proc. Combust. Inst. 30 (2005) 2875-2883

[9] P. A. Fokaides, P. Kasabov, N. Zarzalis, J. Eng. Gas Turbines Power 130 (2008) 011508

[10] H. Li, X. Zhou, J. B. Jeffries, R. K. Hanson, Proc. Combust. Inst. 31 (2007) $3215-3223$

[11] S. Nair, T. Lieuwen, J. Prop. Power 21 (2005) 32-39

[12] T. Yi, E. J. Gutmark, 44th AIAA Aerospace Sciences Meeting and Exhibit, Reno, 2006, AIAA 2006-959

[13] S. Prakash, S. Nair, T. M. Muruganandam, Y. Neumeier, T. Lieuwen, J. M. Seitzman, B. T. Zinn, Proceedings of ASME Turbo Expo, Reno, 2005, GT200568589

[14] B. Böhm, C. Heeger, I. Boxx, W. Meier, A. Dreizler, Proc. Combust. Inst. 32 (2009) 1647-1654

[15] I. Boxx, M. Stöhr, C. Carter, W. Meier, Combust. Flame, accepted

[16] T. M. Muruganandam, J. M. Seitzman, 43rd AIAA Aerospace Sciences Meeting and Exhibit, Reno, 2005, AIAA 2005-1163

[17] P. Weigand, W. Meier, X. R. Duan, W. Stricker, M. Aigner, Combust. Flame 144 (2006) 205-224

[18] W. Meier, X. R. Duan, P. Weigand, Combust. Flame 144 (2006) 225-236

[19] M. Stöhr, R. Sadanandan, W. Meier, Proc. Combust. Inst. 32 (2009) 2925-2932 
[20] Y. Hardalupas, M. Orain, Combust. Flame 139 (2004) 188-207

[21] R. Sadanandan, M. Stöhr, W. Meier, Appl. Phys. B 90 (2008) 609-618

[22] F. Rebosio, A. Widenhorn, B. Noll, M. Aigner, Proceedings of ASME Turbo Expo, Glasgow, 2010, GT2010-22751

[23] J. A. Sutton, J. F. Driscoll, Proc. Combust. Inst. 31 (2007) 1487-1495

[24] P. S. Kothnur, N. T. Clemens, Phys Fluids 17, (2005) 125104

[25] G. Bansal, H. G. Im, Combust. Flame 150 (2007) 404408

[26] S. Nair, T. Lieuwen, J. Prop. Power 23 (2007) 421-427

[27] C. J. Sung, C. K. Law, Proc. Combust. Inst. 26 (1996) 865-873

[28] Y. Ju, H. Guo, K. Maruta, F. Liu, J. Fluid Mech. 342 (1997) 315-334

[29] P. Flohr, J. C. Vassilicos, J. Fluid Mech. 348 (1997) 295-317

[30] K. Bajer, A. P. Bassom, A. D. Gilbert, J. Fluid Mech. 437 (2001) 395-411 